\title{
ASSOCIATION BETWEEN SERUM URIC ACID AND BLOOD GLUCOSE IN YOUNG ADULTS: CROSS-SECTIONAL FINDINGS FROM A BIRTH COHORT
}

Authors: Tulio Loyola Correa ${ }^{1}$, Deise Farias Freitas ${ }^{2}$, Clarice Brinck Brum ${ }^{2}$, Isabel Oliveira de Oliveira $^{2,3}$ (mentor)

${ }^{1}$ Faculty of Medicine, Federal University of Pelotas, Pelotas, Brazil

${ }^{2}$ Centre for Epidemiological Research, Federal University of Pelotas, Pelotas, Brazil

${ }^{3}$ Department of Physiology and Pharmacology, Federal University of Pelotas, Pelotas, Brazil

DOI: https://doi.org/10.26800/LV-142-supp5-55

Introduction:

Serum uric acid (SUA) has been positively associated with diabetes. Most of the studies carried out take into account older adults and there are still few studies in young individuals. Thus, it's necessary to study the association between SUA and glucose in young people to better understand this relationship's trajectory.

Aim:

The aim of the study is to evaluate the association between SUA and blood glucose in 22-year-old young adults.

\section{Materials \& Methods:}

Cross-sectional design with data collected in the 22-year follow-up of the 1993 birth cohort in Pelotas/Brazil. SUA and blood glucose were measured by colorimetric enzymatic assay in blood serum samples. Associations were evaluated by linear regression, considering $p<0.05$, using STATA version 12.0. The analysis was adjusted for total cholesterol, systolic blood pressure, diastolic blood pressure, body mass index, consumption of alcoholic beverages, physical inactivity and smoking.

\section{Results:}

$1.660(47.7 \%)$ males and $1822(52.3 \%)$ females had blood samples collected. The means ( \pm standard deviation) observed in men and women were, respectively: uric acid (mg/dL) [5.2 \pm 1.2 and $3.9 \pm 1.1]$ and glucose $(\mathrm{mg} / \mathrm{dL})$ [91.7 \pm 25.7 and $88.5 \pm 20.9]$. The linear regression coefficient $(95 \% \mathrm{Cl})$ between SUA and glucose was $0.005(0.003 ; 0.008)$ in men and $0.006(0.004 ; 0.009)$ in women; both $p<0.001$. SUA levels $(95 \% \mathrm{Cl})$ according to the glucose tertiles in men and women were, respectively: $5.1(5.0$; $5.2)$ and $3.8(3.7 ; 3.9)$ for the first tertile; $5.2(5.1 ; 5.3)$ and $4.0(3.9 ; 4.0)$ for the second tertile; $5.4(5.3$; $5.5)$ and $4.1(4.0 ; 4.2)$ for the third tertile; with $p<0.001$ for both sexes.

\section{Conclusion:}

SUA presented a positive and significant association with glucose at 22-year-old individuals, regardless of cardiometabolic and behavioral risk factors.

Keywords: Blood Glucose, Diabetes Mellitus, Hyperuricemia, Uric Acid 